\title{
MULTIPLIER TRANSFORMATIONS. III
}

I. I. HIRSCHMAN, JR.

Let $N_{\alpha},-1 / 2<\alpha<1 / 2$, be the space of those complex valued functions $F(n), n \in I$ the additive group of integers, for which $N_{\alpha}[F]$ is finite where

$$
\boldsymbol{N}_{\alpha}[F]=\left[\sum_{-\infty}^{\infty}|F(n)|^{2}(|n|+1)^{2 \alpha}\right]^{1 / 2} .
$$

If $F \in N_{0}$ the Fourier transform

$$
F^{\wedge}(\theta)=\sum_{-\infty}^{\infty} F(n) e^{2 \pi i n \theta}
$$

is defined as a limit in the mean of order 2 for $\theta \in T$, where $T$ is the additive group of real numbers modulo 1 . For such $F$ the following inversion formula is valid,

$$
F(n)=\int_{T} F^{\wedge}(\theta) e^{-2 \pi i n \theta} d \theta .
$$

Let $t(\theta)$ be a bounded measurable function on $T$ and let us define

$$
T F \cdot(n)=\int_{T} F^{\wedge}(\theta) t(\theta) e^{-2 \pi i n \theta} d \theta
$$

for $n \in I$ and $F \in N_{0}$. If

$$
\boldsymbol{N}_{\alpha}[T]=\text { l.u.b. }\left\{\boldsymbol{N}_{\alpha}[T F] / \boldsymbol{N}_{\alpha}[F], F \in \boldsymbol{N}_{\alpha} \cap \boldsymbol{N}_{0}, F \not \equiv 0\right\}
$$

is finite then, since $\boldsymbol{N}_{\alpha} \cap \boldsymbol{N}_{0}$ is dense in $\boldsymbol{N}_{\alpha}, T$ has a unique extension as a bounded linear transformation (with norm $\boldsymbol{N}_{\alpha}[T]$ ) of $\boldsymbol{N}_{\alpha}$ into itself. The problem with which we are concerned is that of finding sufficient conditions on the multiplier function $t(\theta)$ which will insure that the corresponding multiplier transformation $T$ is bounded on $\boldsymbol{N}_{\alpha}$. In the present paper, which continues investigations begun in $[1 ; 2 ; 3]$, we will obtain a sufficient condition involving $\beta$-variation. A function $f(x)$ defined on $I=\{a \leqq x \leqq b\}$ is said to be of bounded $\beta$-variation $(1 \leqq \beta<\infty)$ if $V_{\beta}[f, I]=V_{\beta}[f]$ is finite where

$$
V_{\beta}[f]=\text { l.u.b. }\left[\sum_{k=0}^{n}\left|f\left(x_{k+1}\right)-f\left(x_{k}\right)\right|^{\beta}\right]^{1 / \beta} \text {. }
$$

Received by the editors October 30, 1961. 
Here the least upper bound is taken over all finite sets $a \leqq x_{0}<x_{1}<$ ...< $<x_{n} \leqq b$. Note that if $\beta_{2}>\beta_{1}$

$$
\boldsymbol{V}_{\beta_{2}}[f]^{\beta_{2}} \leqq\left(2\|f\|_{\infty}\right)^{\beta_{2}-\beta_{1}} V_{\beta_{1}}[f]^{\beta_{1}} .
$$

Thus if $V_{\beta_{1}}[f]$ is finite then so is $V_{\beta_{2}}[f]$. Our principal result is that if $V_{\beta}[t(\theta)]$ is finite, $\beta \geqq 2$, then $N_{\alpha}[T]$ is finite for $|\alpha|<1 / \beta$. This result is of interest in that the entire permissible range of $\alpha,-1 / 2<\alpha<1 / 2$, is already obtained for $\beta=2$.

We begin by recalling several results from [2] and [3]. Let $0<\alpha$ $<1 / 2$ be fixed. Note that for $\alpha>0, N_{\alpha} \subset N_{0}$ so that $F^{\wedge}(\theta)$ is well defined for every $F \in N_{\alpha}$. For $t(\theta)$ a bounded measurable function on $T$ we define $\boldsymbol{A}_{\alpha}[t]$ to be the smallest constant such that

$$
\int_{T} \int_{T}\left|F^{\wedge}(\theta)\right|^{2}|t(\theta)-t(\phi)|^{2}|\sin \pi(\theta-\phi)|^{-1-2 \alpha} d \theta d \phi \leqq A_{\alpha}[t] N_{\alpha}[F]^{2}
$$

for every $F \in N_{\alpha} . A_{\alpha}[t]$ may of course be $+\infty$.

Lemma 1. For $0<\alpha<1 / 2$ we have

$$
N_{\alpha}[T] \leqq 2 \operatorname{Max}\left\{\|t\|_{\infty}, A_{\alpha}[t]\right\} .
$$

Lemma 2. For $0<\alpha<1 / 2$ there is a constant $A(\alpha)$ depending only on $\alpha$ such that for any $\psi$ in $T$

$$
\int_{T}|F(\theta)|^{2}|\sin \pi(\theta-\phi)|^{-2 \alpha} d \theta \leqq A(\alpha) N_{\alpha}[F]^{2} .
$$

Lemma 3. Let $f(x)$ be a real function defined on the interval $I=\{a \leqq x \leqq b\}$. For each $\beta>1$ there exists a constant $C(\beta)$ depending only on $\beta$ such that for each $f$ for which $V_{\beta}[f]<\infty$ and each $\epsilon>0$ there exists $f_{\epsilon}(x)$ with the properties:

a. $\left\|f-f_{\epsilon}\right\|_{\infty} \leqq \epsilon \quad x \in I$

b. $V_{1}\left[f_{\epsilon}\right] \leqq C(\beta) V_{\beta}[f]^{\beta} \epsilon^{1-\beta}$.

Here $\|\cdot\|_{\infty}$ is the uniform norm on $I$. This is proved in [2] under the added assumption that $f(x)$ is continuous. However a simple modification of the proof given there shows that this assumption is unnecessary.

Lemma 4. Suppose that $0<\alpha<1 / 2$. Let $t(\theta)$ be of bounded 1-variation on $T$. Then if $T$ is the corresponding multiplier transformation we have

$$
N_{\alpha}[T]^{2} \leqq B(\alpha)\left\{\|t\|_{\infty}^{2}+\|t\|_{\infty} V_{1}[t]\right\}
$$

where $B(\alpha)$ is a finite constant depending only on $\alpha$.

Proof. We begin by proving this result under the assumption that 
$t(\theta)$ is in addition continuous. At the end this restriction will be removed, using a standard approximation argument. For $F \in N_{\alpha}$ consider the quantity

$$
\begin{aligned}
Q & =\int_{T} F^{\wedge}(\theta)^{2} d \theta \int_{T}|t(\theta)-t(\phi)|^{2}|\sin \pi(\theta-\phi)|^{-1-2 \alpha} d \phi \\
& \leqq 2\|t\|_{\infty} \int_{T}\left|F^{\wedge}(\theta)\right|^{2} d \theta \int_{T}|t(\theta)-t(\phi)||\sin \pi(\theta-\phi)|^{-1-2 \alpha} d \phi .
\end{aligned}
$$

We have

$$
\int_{T}|t(\theta)-t(\phi)||\sin \pi(\theta-\phi)|^{-1-2 \alpha} d \phi \leqq I_{1}+I_{2}
$$

where

$$
\begin{aligned}
& I_{1}=\int_{\theta}^{\theta+1 / 2}|\sin \pi(\theta-\phi)|^{-1-2 \alpha} d \phi \int_{\theta}^{\phi}|d t(\psi)|, \\
& I_{2}=\int_{\theta-1 / 2}^{\theta}|\sin \pi(\theta-\phi)|^{-1-2 \alpha} d \phi \int_{\phi}^{\theta}|d t(\psi)| .
\end{aligned}
$$

By Fubini's theorem

$$
I_{1}=\int_{\theta}^{\theta+1 / 2}|d t(\psi)| \int_{\psi}^{\theta+1 / 2}|\sin \pi(\theta-\phi)|^{-1-2 \alpha} d \phi .
$$

An easy computation shows that there exists a constant $A_{1}(\alpha)$ such that if $\theta \leqq \psi \leqq \theta+1 / 2$

$$
\int_{\psi}^{\theta+1 / 2}|\sin \pi(\theta-\phi)|^{-1-2 \alpha} d \phi \leqq A_{1}(\alpha)|\sin \pi(\theta-\psi)|^{-2 \alpha} .
$$

Thus

$$
I_{1} \leqq A_{1}(\alpha) \int_{\theta}^{\theta+1 / 2}|\sin \pi(\theta-\psi)|^{-2 \alpha}|d t(\psi)|,
$$

and similarly

$$
I_{2} \leqq A_{1}(\alpha) \int_{\theta-1 / 2}^{\theta}|\sin \pi(\theta-\psi)|^{-2 \alpha} d t(\psi) .
$$

Making use of these inequalities and using Fubini's theorem we find that

$$
\begin{aligned}
Q & \leqq 2 A_{1}(\alpha)\|t\|_{\infty} \int_{T}\left|F^{\wedge}(\theta)\right|^{2} d \theta \int_{T}|\sin \pi(\theta-\psi)|-2 \alpha|d t(\psi)| \\
& \leqq 2\|t\|_{\infty} A_{1}(\alpha) \int_{T}|d t(\psi)| \int_{T}\left|F^{\wedge}(\theta)\right|^{2}|\sin \pi(\theta-\psi)|^{-2 \alpha} d \theta .
\end{aligned}
$$


Applying Lemma 2 we obtain

$$
Q \leqq 2 A_{1}(\alpha) A(\alpha)\|t\|_{\infty} V_{1}[t] N_{\alpha}[F]^{2} .
$$

Thus $\boldsymbol{A}_{\alpha}[t] \leqq 2 A_{1}(\alpha) A(\alpha)\|t\|_{\infty} V_{1}[t]$. Our proof is now complete if $t(\theta)$ is continuous. If $t(\theta)$ is not continuous we set

$$
t_{n}(\theta)=\int_{T} k_{n}(\theta-\phi) t(\phi) d \phi \quad n=1,2, \cdots
$$

where $k_{n}(\theta)$ is any sequence of functions on $T$ satisfying:

i. $k_{n}(\theta)$ is continuous;

ii. $k_{n}(\theta) \geqq 0, \int_{T} k_{n}(\theta) d \theta=1$;

iii. $\lim _{n \rightarrow \infty} \int_{U} k_{n}(\theta) d \theta=1$, for any fixed open set $U$ in $T$ which contains 0 .

With these assumptions it is easily verified that:

i. $\left\|t_{n}\right\|_{\infty} \leqq\|t\|_{\infty}$;

ii. $V_{1}\left[t_{n}\right] \leqq V_{1}[t]$;

iii. $\lim _{n \rightarrow \infty} t_{n}(\theta)=t(\theta)$ for all $\theta$ at which $t(\cdot)$ is continuous.

Let $T_{n}$ be the multiplier transform generated by $t_{n}(\theta)$; then

$$
N_{\alpha}[T] \leqq \liminf _{n \rightarrow \infty} N_{\alpha}\left[T_{n}\right]
$$

Since $t_{n}(\theta)$ is continuous

$$
\begin{aligned}
N_{\alpha}\left[T_{n}\right]^{2} & \leqq B(\alpha)\left\{\left\|t_{n}\right\|_{\infty}^{2}+\left\|t_{n}\right\|_{\infty} V_{1}\left[t_{n}\right]\right\}, \\
& \leqq B(\alpha)\left\{\|t\|_{\infty}^{2}+\|t\|_{\infty} V_{1}[t]\right\} .
\end{aligned}
$$

Combining these results our desired lemma follows.

THEOREM. Let $t(\theta)$ be defined for $\theta \in T$ and let $T$ be the corresponding multiplier transformation. If $V_{\beta}[t]$ is finite (where $\beta>2$ ) then

$$
N_{\alpha}[T]<\infty \quad \text { if }|\alpha|<1 / \beta .
$$

Proof. By Lemma 3 there exists a sequence of functions $s_{n}(\theta)$, $\theta \in T$, such that

$$
\begin{aligned}
\left\|s_{n}-t\right\|_{\infty} & \leqq 2^{-n} \\
V_{1}\left[s_{n}\right] & \leqq C(\beta) V_{\beta}[f]^{\beta 2^{n(\beta-1)}} \quad n=1,2, \cdots .
\end{aligned}
$$

Let

$$
\begin{aligned}
& t_{1}(\theta)=s_{1}(\theta), \\
& t_{n}(\theta)=s_{n}(\theta)-s_{n-1}(\theta)
\end{aligned} \quad n=2,3, \cdots .
$$


Then

$$
t(\theta)=\sum_{1}^{\infty} t_{n}(\theta),
$$

and thus by an evident argument

$$
N_{\alpha}[T] \leqq \sum_{1}^{\infty} N_{\alpha}\left[T_{n}\right]
$$

where $T_{n}$ is the multiplier transformation generated by the multiplier function $t_{n}(\theta)$. We have

$$
\begin{aligned}
\left\|t_{n}\right\|_{\infty} & =O\left(2^{-n}\right) & & n=1,2, \cdots, \\
V_{1}\left[t_{n}\right] & =O\left(2^{n(\beta-1)}\right) & n & =1,2, \cdots .
\end{aligned}
$$

Choose $\gamma, \alpha<\gamma<1 / 2$. By Lemma 4

$$
\begin{aligned}
N_{\gamma}\left[T_{n}\right] & =O\left[\left(2^{-n}\right)^{2}+2^{-n} 2^{n(\beta-1)}\right]^{1 / 2} \\
& =O\left(2^{n(\beta / 2-1)}\right) .
\end{aligned}
$$

On the other hand by Parseval's equality

$$
N_{0}\left[T_{n}\right]=\left\|t_{n}\right\|_{\infty}=O\left(2^{-n}\right) .
$$

Applying the Riesz-Thorin convexity theorem we find that if $\alpha=(1-\theta) 0+\theta \gamma$ then

$$
\begin{aligned}
N_{\alpha}\left[T_{n}\right] & =O\left(2^{-n(1-\theta)} 2^{n(\beta / 2-1) \theta}\right), \\
& =O\left(2^{n(-1+\beta \alpha / 2 \gamma)}\right) .
\end{aligned}
$$

Thus the series $\sum_{1}^{\infty} N_{\alpha}\left[T_{n}\right]$ is convergent if $\beta \alpha / 2 \gamma<1$; that is if $\alpha<2 \gamma / \beta$. Since $\gamma$ is arbitrary subject to the restriction $\alpha<\gamma<1 / 2$, it is always possible to choose $\gamma$ so that $\alpha<2 \gamma / \beta$ if $0<\alpha<1 / \beta$. Thus our theorem is true if $0<\alpha<1 / \beta$. The case $-1 / \beta<\alpha<0$ follows by a familiar duality argument, while the case $\alpha=0$ is trivial.

For $f(x)$ defined on the interval $I$ let $W_{\beta}[f, I]$ be the smallest constant such that for every $\epsilon>0$ there exists a function $f_{\epsilon}(x), x \in I$, satisfying:

a. $\left\|f-f_{\epsilon}\right\|_{\infty} \leqq \epsilon$,

b. $V_{1}\left[f_{\epsilon}, I\right] \leqq W_{\beta}[f, I] \epsilon^{1-\beta}$.

$W_{\beta}[f, I]$ can of course be $+\infty$. Lemma 3 asserts that

$$
W_{\beta}[f, I] \leqq C(\beta) V_{\beta}[f, I]^{\beta} .
$$

The assumption in our principal theorem that $V_{\beta}[f, T]<\infty$ is made only to insure that $W_{\beta}[f, T]<\infty$. The following lemma shows that 
the assumption $W_{\beta}[f, T]<\infty$ is "almost" as strong as the assumption $V_{\beta}[f, T]<\infty$.

Lemma 5. For each $\beta, 1 \leqq \beta<\infty$, and each $\gamma>\beta$ there exists a finite constant $A(\beta, \gamma)$ such that

$$
V_{\gamma}[f, I] \leqq A(\beta, \gamma)\|f\|_{\infty}^{(\gamma-\beta) / \gamma} W_{\beta}[f, I]^{1 / \gamma} .
$$

Proof. For each $k=0,1, \cdots$ let $f_{k}$ satisfy

$$
\begin{aligned}
\left\|f-f_{k}\right\|_{\infty} & \leqq 2^{-k}\|f\|_{\infty}, \\
V_{1}\left[f_{k}\right] & \leqq W_{\beta}[f] 2^{k(\beta-1)}\|f\|_{\infty}^{1-\beta} .
\end{aligned}
$$

If we define

$$
\begin{aligned}
& g_{0}(x)=f_{0}(x), \\
& g_{k}(x)=f_{k}(x)-f_{k-1}(x) \quad k=1,2, \cdots,
\end{aligned}
$$

then

$$
\sum_{k=0}^{\infty} g_{k}(x)=f(x) \quad x \in I
$$

Moreover

$$
\begin{aligned}
\left\|g_{k}(x)\right\|_{\infty} & \leqq 42^{-k}\|f\|_{\infty}, \\
V_{1}\left[g_{k}\right] & \leqq 2 W_{\beta}[f] 2^{k(\beta-1)}\|f\|_{\infty}^{1-\beta} .
\end{aligned}
$$

It is easy to see using Hölder's inequality that $V_{\gamma}[f] \leqq \sum_{0}^{\infty} V_{\gamma}\left[g_{k}\right]$. Also

$$
V_{\gamma}[g]^{\gamma} \leqq\left(2\|g\|_{\infty}\right)^{\gamma-1} V_{1}[g]
$$

Thus

$$
\begin{aligned}
V_{\gamma}[f] & \leqq \sum_{0}^{\infty}\left(2^{3-k}\|f\|_{\infty}\right){ }^{(\gamma-1) / \gamma}\left(2 W_{\beta}[f] 2^{k(\beta-1)}\|f\|_{\infty}^{1-\beta}\right)^{1 / \gamma} \\
& \leqq\|f\|_{\infty}^{(\gamma-\beta) / \gamma} W_{\beta}[f]^{1 / \gamma} 2^{(3 \gamma-2) / \gamma} \sum_{0}^{\infty} 2^{-k(\gamma-\beta) / \gamma} \\
& \leqq A(\beta, \gamma)\|f\|_{\infty}^{(\gamma-\beta) / \gamma} W_{\beta}[f]^{1 / \gamma} .
\end{aligned}
$$

On the other hand the assumption $W_{\beta}[f]<\infty$ is slightly weaker than the assumption $V_{\beta}[f]<\infty$ in that for $\beta>1$ no inequality of the form

$$
V_{\beta}[f, I]^{\beta} \leqq A^{\prime}(\beta) W_{\beta}[f, I]
$$


is true for all $f$. To see this let us set $I_{k}=\{k-1 \leqq x \leqq k\}$ for all $k=0,1,2, \cdots$, and $I^{N}=I_{1} \cup I_{2} \cup \cdots \cup I_{N}$. Further let

$$
f_{1}(x)=2^{-k / \beta} \sin \left[2^{k}(2 \pi x)\right] \quad x \in I_{k} .
$$

Very simple computations show that there are positive constants $c_{1}(\beta)$ and $c_{2}(\beta)$ independent of $k$ and $N$ such that

$$
\begin{array}{ll}
W_{\beta}\left[f_{1}, I_{k}\right] \geqq c_{1}(\beta) & k=1,2, \cdots, \\
W_{\beta}\left[f_{1}, I^{N}\right] \leqq c_{2}(\beta) & N=1,2, \cdots .
\end{array}
$$

It is evident that

$$
\sum_{1}^{N} V_{\beta}\left[f, I_{k}\right]^{\beta} \leqq V_{\beta}\left[f, I^{N}\right]^{\beta} .
$$

If (3) held then using (1) we would have

$$
C(\beta)^{-1} \sum_{1}^{N} W_{\beta}\left[f, I_{k}\right] \leqq A^{\prime}(\beta) W_{\beta}\left[f, I^{N}\right] .
$$

However for $f=f_{1}$ and for $N$ sufficiently large this is impossible.

\section{REFERENCES}

1. A. Devinatz and I. I. Hirschman, Jr., Multiplier transformations on $1^{2, \alpha}$, Ann. of Math. 69 (1959), 575-587.

2. I. I. Hirschman, Jr., On multiplier transformations, Duke Math. J. 26 (1959), 221-242.

3. —-, Multiplier transformations. II, Duke Math. J. 28 (1961), 45-56.

WASHINGTON UNIVERSITY 